\title{
CONTROVERSY
}

\section{Extending preimplantation genetic diagnosis: medical and non-medical uses}

J A Robertson

J Med Ethics 2003;29:213-216

New uses of preimplantation genetic diagnosis (PGD) to screen embryos prior to transfer raise ethical, legal, and policy issues that deserve close attention. Extensions for medical purposes, such as to identify susceptibility genes, late onset disease, and human leukocyte antigen (HLA) matching, are usually ethically acceptable. Whether embryo screening for gender, perfect pitch, or other non-medical characteristics are also acceptable depends upon the parental needs served and the harm posed to embryos, children, and society. Speculations about potential future uses of PGD should not prevent otherwise acceptable current uses of PGD.

Correspondence to: Professor J A Robertson, Law School, University of Texas, 727 Dean Keeton Street, Austin, Texas, USA; jrobertson@ mail.law.utexas.edu

Accepted 28 October 2002
D ebate about new reproductive technologies often cites preimplantation genetic diagnosis (PGD) - the technique by which early human embryos are genetically screened for selection for transfer to the uterus-as a practice that needs close ethical, legal, and social scrutiny. The use of PGD is growing, as are the indications for it. This article describes medical and nonmedical extensions of PGD, and discusses the ethical, legal, and policy issues which they raise.

\section{PGD AND ITS PREVALENCE}

PGD has been available since 1990 for testing of aneuploidy in low prognosis in vitro fertilisation (IVF) patients, and for single gene and X linked diseases in at risk couples. One cell (blastomere) is removed from a cleaving embryo and tested for the genetic or chromosomal condition of concern. Some programmes analyse polar bodies extruded from oocytes during meiosis, rather than blastomeres. ${ }^{1}$ Cells are then either karyotyped to identify chromosomal abnormalities, or analysed for single gene mutations and linked markers.

Physicians have performed more than 3000 clinical cycles of PGD since 1990, with more than 700 children born as a result. The overall pregnancy rate of $24 \%$ is comparable to assisted reproductive practices which do not involve embryo or polar body biopsy. ${ }^{1}$ Four centres (Chicago, Livingston (New Jersey), Bologna, and Brussels) accounted for nearly all the reported cases. More than 40 centres worldwide offer the procedure, however, including other centres in the United States and Europe, four centres in London and centres in the eastern Mediterranean, Southeast Asia, and Australia.

More than two thirds of PGD has occurred to screen out embryos with chromosomal abnormalities in older IVF patients and in patients with a history of miscarriage. About 1000 cycles have involved single gene mutational analysis. ${ }^{1}$ Mutational analysis requires additional skills beyond karyotyping for aneuploidies, including the ability to conduct the mutliplex polymerase chain reaction (PCR) of the gene of interest and related markers.

Several new indications for PGD single gene mutational analysis have recently been reported. New uses include PGD to detect mutations for susceptibility to cancer and for late onset disorders such as Alzheimer's disease. ${ }^{23}$ In addition, parents with children needing hematopoietic stem cell transplants have used PGD to ensure that their next child is free of disease and a good tissue match for an existing child. ${ }^{4}$ Some persons are also requesting PGD for gender selection for both first and later born children, and others have speculated that selection of embryos for a variety of non-medical traits is likely in the future.

PGD is ethically controversial because it involves the screening and likely destruction of embryos, and the selection of offspring on the basis of expected traits. While persons holding right to life views will probably object to PGD for any reason, those who view the early embryo as too rudimentary in development to have rights or interests see no principled objection to all PGD. They may disagree, however, over whether particular reasons for PGD show sufficient respect for embryos and potential offspring to justify intentional creation and selection of embryos. Donation of unwanted embryos to infertile couples reduces this problem somewhat, but there are too few such couples to accept all unwanted embryos, and in any event, the issue of selecting offspring traits remains.

Although ethical commentary frequently mentions PGD as a harbinger of a reproductive future of widespread genetic selection and alteration of prospective offspring, its actual impact is likely to be quite limited. ${ }^{5}{ }^{6}$ Even with increasing use the penetrance of PGD into reproductive practice is likely to remain a very small percentage of the 150000 plus cycles of IVF performed annually throughout the world. Screening for susceptibility and late onset diseases is limited by the few diseases for which single gene predispositions are known. Relatively few parents will face the need to conceive another child to provide an existing child with matched stem cells. Nor are nonmedical uses of PGD, other than for gender, likely to be practically feasible for at least a decade or more. Despite the limited reach of PGD, the ethical, legal, and policy issues that new uses raise, deserve attention. 


\section{NEW MEDICAL USES}

New uses of PGD may be grouped into medical and non-medical categories. New medical uses include not only screening for rare Mendelian diseases, but also for susceptibility conditions, late onset diseases, and HLA matching for existing children.

Embryo screening for susceptibility and late onset conditions are logical extensions of screening for serious Mendelian diseases. For example, using PGD to screen out embryos carrying the $\mathrm{p} 53$ or BRCAl\&2 mutations prevent the birth of children who would face a greatly increased lifetime risk of cancer, and hence require close monitoring, prophylactic surgery, or other preventive measures. PGD for highly penetrant adult disorders such as Alzheimer's or Huntington's disease prevents the birth of a child who will be healthy for many years, but who in her late 30s or early 40 s will experience the onset of progressive neurological disease leading to an early death.

Although these indications do not involve diseases that manifest themselves in infancy or childhood, the conditions in question lead to substantial health problems for offspring in their thirties or forties. ${ }^{7}$ Avoiding the birth of children with those conditions thus reflects the desire of parents to have offspring with good prospects for an average life span. If PGD is accepted to exclude offspring with early onset genetic diseases, it should be accepted for later onset conditions as well.

PGD for adult onset disorders does mean that a healthy child might then be born to a person with those conditions who is likely to die or become incompetent while the child is dependent on her. ${ }^{8}$ But that risk has been tolerated in other cases of assisted reproduction, such as intrauterine insemination with sperm of a man who is HIV positive, IVF for women with cystic fibrosis, and use of gametes stored prior to cancer therapy. As long as competent caregivers will be available for the child, the likely death or disability of a parent does not justify condemning or stopping this use, anymore than that reproduction by men going off to war should be discouraged.

A third new medical indication-HLA matching to an existing child-enables a couple to have their next child serve as a matched hematopoietic stem cell donor for an existing sick child. It may also ensure that the new child does not also suffer from that same disease. The availability of PGD, however, should not hinge on that fact, as the Human Fertilisation and Embryology Authority, in the UK, now requires. ${ }^{9}$ A couple that would coitally conceive a child to be a tissue donor should be free to use PGD to make sure that that child will be a suitable match, regardless of whether that child is also at risk for genetic disease. Parents who choose PGD for this purpose are likely to value the new child for its own sake, and not only for the stem cells that it will make available. They do not use the new child as a "mere means" simply because they have selected HLA matched embryos for transfer. ${ }^{10}{ }^{11}$

\section{NON-MEDICAL USES OF PGD}

More ethically troubling has been the prospect of using PGD to screen embryos for genes that do not relate to the health of resulting children or others in the family. Many popular accounts of PGD assume that it will eventually be used to select for such non-medical traits as intelligence, height, sexual orientation, beauty, hair and eye colour, memory, and other factors. ${ }^{56}$ Because the genetic basis of those traits is unknown, and in any case is likely to involve many different genes, they may not be subject to easy mutational analysis, as Mendelian disease or susceptibility conditions are. Aside from gender, which is identifiable through karyotyping, it is unrealistic to think that non-medical screening for other traits, with the possible exception of perfect pitch, will occur anytime soon.
Still, it is useful to consider the methodology that ethical assessment of non-medical uses of PGD, if available, should follow. The relevant questions would be whether the proposed use serves valid reproductive or rearing interests; whether those interests are sufficient to justify creating and destroying embryos; whether selecting for a trait will harm resulting children; whether it will stigmatise existing persons, and whether it will create other social harms.

To analyse how these factors interact, I discuss PGD for sex selection and for children with perfect pitch. Similar issues would arise with PGD for sexual orientation, for hair and eye color, and for intelligence, size, and memory.

\section{PGD for gender selection}

The use of medical technology to select the sex of offspring is highly controversial because of the bias against females which it usually reflects or expresses, and the resulting social disruptions which it might cause. PGD for gender selection faces the additional problem of appearing to be a relatively weak reason for creating and selecting embryos for discard or transfer.

The greatest social effects of gender selection arise when the gender of the first child is chosen. Selection for first children will overwhelmingly favour males, particularly if one child per family population policies apply. If carried out on a large scale, it could lead to great disparities in the sex ratio of the population, as has occurred in China and India through the use of ultrasound screening and abortion. ${ }^{12}{ }^{13}$ PGD, however, is too expensive and inaccessible to be used on a wide scale for sex selection purposes. Allowing it to be used for the first child is only marginally likely to contribute to societal sex ratio imbalances. But its use is likely to reflect cultural notions of male privilege and may reinforce entrenched sexism toward women.

The use of PGD to choose a gender opposite to that of an existing child or children is much less susceptible to a charge of sexism. Here a couple seeks variety or "balance" in the gender of offspring because of the different rearing experiences that come with rearing children of different genders. Psychologists now recognise many biologically based differences between male and female children, including different patterns of aggression, learning, and spatial recognition, as well as hormonal differences. ${ }^{14}{ }^{15}$ It may not be sexist in itself to wish to have a child or children of each gender, particularly if one has two or more children of the same gender.

Some feminists, however, would argue that any attention to the gender of offspring is inherently sexist, particularly when social attitudes and expectations play such an important role in constructing sex role expectations and behaviours. ${ }^{16}$ Other feminists find the choice of a child with a gender different from existing children to be morally defensible as long as "the intention and consequences of the practice are not sexist", which is plausibly the case when gender variety in children is sought. ${ }^{17}$ Desiring the different rearing experiences with boys and girls does not mean that the parents, who have already had children of one gender, are sexists or likely to value unfairly one or the other gender. ${ }^{18}$

Based on this analysis the case is weak for allowing PGD for the first child, but may be acceptable for gender variety in a family. With regard to the first child, facilitating preferences for male firstborns carries a high risk of promoting sexist social mores. It may also strike many persons as too trivial a concern to meet shared notions of the special respect due preimplantation embryos. A proponent of gender selection, however, might argue that cultural preferences for firstborn males should be tolerated, unless a clearer case of harm has been shown. If PGD is not permitted, pregnancy and abortion might occur instead.

The case for PGD for gender variety is stronger because the risk of sexism is lessened. A couple would be selecting the gender of a second or subsequent children for variety in rearing experiences, and not out of a belief that one gender is 
privileged over another. Gender selection in that case would occur without running the risks of fostering sexism and hurting women. ${ }^{18}$

The question still arises whether the desire for gender variety in children, even if not sexist, is a strong enough reason to justify creating and discarding embryos. The answer depends on how strong an interest that is. No one has yet marshalled the evidence showing that the need or desire for gender variety in children is substantial and important, or whether many parents would refrain from having another child if PGD for gender variety were not possible. More evidence of the strength and prevalence of this need would help in reaching a conclusion. If that case is made, then PGD for gender variety might be acceptable as well. ${ }^{19}$

The ethics committee of the American Society of Reproductive Medicine (ASRM) has struggled with these issues in a series of recent opinions. It initially addressed the issue of PGD for gender selection generally, and found that it "should be discouraged" for couples not going through IVF, and "not encouraged" for couples who were, but made no distinction between PGD for gender selection of first and later children. ${ }^{20}$ Subsequently, it found that preconception gender selection would be acceptable for purposes of gender variety but not for the first child. ${ }^{18}$

Perceiving these two positions to be inconsistent, a doctor who wanted to offer PGD for gender selection inquired of the ethics committee why preconception methods for gender variety, which lacked $100 \%$ certainty, were acceptable but PGD, which guaranteed that certainty, was not. Focusing only on the sexism and gender discrimination issue, the chair of the ethics committee, in a widely publicised letter, found that PGD for gender balancing would be acceptable. ${ }^{21}$ When the full committee reconsidered the matter, it concluded that it had not yet received enough evidence that the need for gender variety was so important in families that it justified creating and discarding embryos for that purpose. ${ }^{19}$ In the future if such evidence was forthcoming then PGD for gender variety might also be acceptable.

What might constitute such evidence? One source would be families with two or more children of one gender who very much would like to have another child but only if they could be sure that it would be a child of the gender opposite of existing children. Given the legitimacy of wanting to raise children of both genders, reasonable persons might find that this need outweighs the symbolic costs of creating and discarding embryos for that purpose.

Another instance would be a case in which a couple has had a girl, but now wants a boy in order to meet cultural norms of having a male heir or a male to perform funeral rituals or play other cultural roles. An IVF programme in India is now providing PGD to select male offspring as the second child of couples who have already had a daughter. ${ }^{22}$ Because of the importance of a male heir in India, those couples might well consider having an abortion if pregnant with a female fetus (even though illegal in India for that purpose). In that setting PGD for gender selection for gender variety appears to be justified.

\section{PGD for perfect pitch}

Perfect or "absolute" pitch is the ability to identify and recall musical notes from memory. ${ }^{23}$ Although not all great or successful musicians have perfect pitch, a large number of them do. Experts disagree over whether perfect pitch is solely inborn or may also be developed by early training, though most agree that a person either has it or does not. It also runs in families, apparently in an autosomal dominant pattern. ${ }^{23}$ The gene or genes coding for this capacity have not, however, been mapped, much less sequenced. Because genes for perfect pitch may also relate to the genetic basis for language or other cognitive abilities, research to find that gene may be forthcoming.
Once the gene for perfect pitch or its linked markers are identified, it would be feasible to screen embryos for those alleles, and transfer only those embryos that test positive. The prevalence of those genes is quite low (perhaps three in 100) in the population, but high in certain families. ${ }^{23}$ Thus only persons from those families who have a strong interest in the musical ability of their children would be potential candidates for PGD for perfect pitch. Many of them are likely to take their chances with coital conception and exposure of the child to music at an early age. Some couples, however, may be willing to undergo IVF and PGD to ensure musical ability in their child. Should their request be accepted or denied?

As noted, the answer to this question depends on the importance of the reproductive choice being asserted, the burdens of the selection procedure, its impact on offspring, and its implications for deselected groups and society generally. The strongest case for the parents is if they persuasively asserted that they would not reproduce unless they could select that trait, and they have a plausible explanation for that position. Although the preference might appear odd to some, it might also be quite understandable in highly musical families, particularly ones in which some members already have perfect pitch. Parents clearly have the right to instill or develop a child's musical ability after birth. They might reasonably argue that they should have that right before birth as well.

If so, then creating and discarding embryos for this purpose should also be acceptable. If embryos are too rudimentary in development to have inherent rights or interests, then no moral duty is violated by creating and destroying them. ${ }^{24}$ Some persons might think that doing so for trivial or unimportant reasons debases the inherent dignity of all human life, but having a child with perfect pitch will not seem trivial to parents seeking this technique. Ultimately, the judgment of triviality or importance of the choice within a broad spectrum rests with the couple. If they have a strong enough preference to seek PGD for this purpose and that preference rationally relates to understandable reproductive goals, then they have demonstrated its great importance to them. Only in cases unsupported by a reasonable explanation of the need-for example, perhaps creating embryos to pick eye or hair colour, should a person's individual assessment of the importance of creating embryos be condemned or rejected.

A third relevant factor is whether musical trait selection is consistent with respect for the resulting child. Parents who are willing to undergo the costs and burdens of IVF and PGD to have a child with perfect pitch may be so overly invested in the child having a musical career that they will prevent it from developing its own personality and identity. Parents, however, are free to instill and develop musical ability once the child is born, just as they are entitled to instill particular religious views. It is difficult to say that they cross an impermissible moral line of risk to the welfare of their prospective child in screening embryos for this purpose. Parents are still obligated to provide their child with the basic education and care necessary for any life plan. Wanting a child to have perfect pitch is not inconsistent with parents also wanting their child to be well rounded and equipped for life in other contexts.

A fourth factor, impact on deselected groups, is much less likely to be an issue in the case of perfect pitch because there is no stigma or negative association tied to persons without that trait. Persons without perfect pitch suffer no stigma or opprobrium by the couple's choice or public acceptance of it, as is arguably the case with embryo selection on grounds of gender, sexual orientation, intelligence, strength, size, or other traits. Nor is PGD for perfect pitch likely to perpetuate unfair class advantages, as selection for intelligence, strength, size, or beauty might.

A final factor is the larger societal impact of permitting embryo screening for a non-medical condition such as perfect pitch. A valid concern is that such a practice might then legitimise embryo screening for other traits as well, thus moving us toward a future in which children are primarily valued 
according to the attractiveness of their expected characteristics. But that threat is too hypothetical to justify limiting what are otherwise valid exercises of parental choice. It is highly unlikely that many traits would be controlled by genes that could be easily tested in embryos. Gender is determined by the chromosome, and the gene for pefect pitch, if ever found, would be a rare exception to the multifactorial complexity of such traits. Screening embryos for perfect pitch, if otherwise acceptable, should not be stopped simply because of speculation about what might be possible several decades from now.

\section{PGD for other non-medical traits}

The discussion of PGD for perfect pitch illustrates the issues that would arise if single gene analysis became possible for other traits, such as sexual orientation, hair or eye colour, or height, intelligence, size, strength, and memory. In each case the ethical assessment depends on an evaluation of the importance of the choice to the parents and whether that choice plausibly falls within societal understandings of parental needs and choice in reproducing and raising children. If so, it should usually be a sufficient reason to create and screen embryos. The effect on resulting offspring would also be of key moral importance. Whether selection carries a public or social message about the worth of existing groups should also be addressed.

Applying this methodology might show that some instances of non-medical selection are justified, as we have seen with embryo selection for gender variety and perhaps for having a child with perfect pitch. The acceptability of PGD to select other non-medical traits will depend on a careful analysis of the relevant ethical factors, and social acceptance of much greater parental rights to control the genes of offspring than now exists.

\section{CONCLUSION}

Although new indications are emerging for PGD, it is likely to remain a small part of reproductive practice for some time to come. Most new indications serve legitimate medical purposes, such as screening for single gene mutations for late onset disorders or susceptibility to cancer. There is also ethical support for using PGD to assure that a child is an HLA match with an existing child.

More controversial is the use of PGD to select gender or other non-medical traits. As with medical uses, the acceptability of non-medical screening will depend upon the interests served and the effects of using PGD for those purposes. Speculations about potential future non-medical uses should not restrict new uses of PGD which are otherwise ethically acceptable.

\section{ACKNOWLEDGEMENT}

Professor Robertson was supported by the Ethical, Legal, and Social Issues Program of the National Institute of Human Genome Research, US National Institutes of Health (USA).

\section{REFERENCES}

1 International Working Group on Preimplantation Genetics. Preimplantation genetic diagnosis: experience of 3000 clinical cycles. Report of the 11th annual meeting, May 15, 2001. Reprod Biomedicine Online 2001;3:49-53.

2 Verlinsky Y, Rechitsky S, Verlinsky O, et al. Preimplantation diagnosis of P53 tumor suppressor gene mutations. Reprod Biomedicine Online 2001;2:102-5

3 Verlinsky Y, Rechitsky S, Schoolcraft W, et al. Preimplantation diagnosis for fanconi anemia combined with HLA matching. JAMA 2001;285:3130-3

4 Verlinsky Y, Rechitsky S, Verlinsky $O$, et al. Preimplantation diagnosis for early-onset alzheimer's disease caused by V717L mutation. JAMA 2002; 283:1018-21.

5 Fukuyama F. Our postmodern future: consequences of the biotechnology revolution. New York: Farrar, Strauss, \& Giroux, 2002 6 Stock G. Redesigning humans: our inevitable genetic future. New York: Houghton Mifflin, 2002

7 Simpson JL. Celebrating preimplantation genetic diagnosis of p53 mutations in Li-Fraumeni syndrome. Reprod Biomedicine Online 2001;3: $2-3$

8 Towner D, Loewy RS. Ethics of preimplantation diagnosis for a woman destined to develop early-onset alzheimer disease. JAMA 2002;283: 1038-40.

9 Human Fertilisation and Embryology Authority. Opinion of the ethics committee. Ethical issues in the creation and selection of preimplantation embryos to produce tissue donors. London: HFEA, 2001 Nov 22.

10 Pennings G, Schots S, Liebaers I. Ethical considerations on preimplantation genetic diagnosis for HLA typing to match a future child as a donor of haematopoietic stem cells to a sibling. Hum Reprod 2002;17:534-8

11 Robertson JA, Kahn J,Wagner J. Conception to obtain hematopoietic stem cells. Hastings Cent Rep 2002;32:34-40.

12 Sen A. More than 100 million women are missing. New York Review of Books 1990;37:61-8.

13 Eckholm E. Desire for sons drives use of prenatal scans in China. The New York Times 2002 Jun 21: A3

14 Jaccoby EE, Jacklin CN. The psychology of sex differences. Palo Alto: Stanford University Press, 1974.

15 Robertson JA. Preconception gender selection. Am J Bioeth 2001;1:2-9.

16 Grubb A, Walsh P. Gender-vending II. Dispatches 1994;1:1-3.

17 Mahowald MB. Genes, women, equality. New York: Oxford University Press, 2000: 121

18 American Society of Reproductive Medicine, Ethics Committee. Preconception gender selection for nonmedical reasons. Fertil Steril 2001;75:861-4.

19 Robertson JA. Sex selection for gender variety by preimplantation genetic diagnosis. Fert Steril 2002;78:463.

20 American Society of Reproductive Medicine, Ethics Committee. Sex selection and preimplantation genetic diagnosis. Fertil Steril 1999;72:595-8.

21 Kolata G. Society approves embryo selection. The New York Times 2001 Sept 26: A14.

22 Malpani A, Malpani A, Modi D. Preimplantation sex selection for family balancing in India. Hum Reprod 2002;17:11-12.

23 Blakeslee S. Perfect pitch: the key may lie in the genes. The New York Times 1990 Nov 30: 1.

24 American Society of Reproductive Medicine, Ethics Committee. Ethical considerations of assisted reproductive technologies. Fertil Steril 1994;62(suppl):32-7S 